duration was not documented. DAPT was excessive in $40 \%$ and insufficient in $8 \%$. Planned DAPT duration was documented at time of angiography or prior to discharge in $80 \%$. Where documented, 12 months DAPT was advised for $92.5 \%$ overall and $93.3 \%$ following elective PCI. Proton-Pump Inhibitors were not prescribed in $44 \%$.

Within 12 months of commencing DAPT, 46 of 50 patients (92\%) were reviewed in outpatient clinic. At clinic, planned date of DAPT discontinuation was documented for 35 patients (70\%). Among patients who received excessive DAPT, one patient required hospital admission with a TIMI grade minor bleed, 1 patient had a TIMI grade minimal bleed and 1 patient's urgent prostate biopsy was unnecessarily delayed.

Conclusions/Implications Our results indicate that current DAPT prescribing procedures in our department are ineffective and not adhering to guidelines. This could put patients at unnecessary risk of bleeding and ischaemic events. A standardised protocol-based approach to DAPT may improve this practice and reduce risk for our patients.

\section{ANTITHROMBOTIC THERAPY FOLLOWING SURGICAL REVASCULARISATION IN NON ST-ELEVATION MYOCARDIAL INFARCTION (NSTEMI)- WHAT IS THE OPTIMAL REGIME?}

${ }^{1} \mathrm{~J}$ Mailey, ${ }^{1} \mathrm{C}$ Shannon, ${ }^{2} \mathrm{~L}$ Burns, ${ }^{1} \mathrm{P}$ Brennan, ${ }^{3} \mathrm{~N}$ Johnston. ${ }^{1}$ Royal Victoria Hospital, Belfast, UK; ${ }^{2}$ Southern Trust, UK; ${ }^{3}$ Belfast Health and Social Care Trust, Belfast, UK

\subsection{6/heartjnl-2020-ICS.60}

Background Current European Society of Cardiology (ESC) guidelines advocate the use of dual antiplatelet therapy (DAPT) following surgical revascularisation of patients

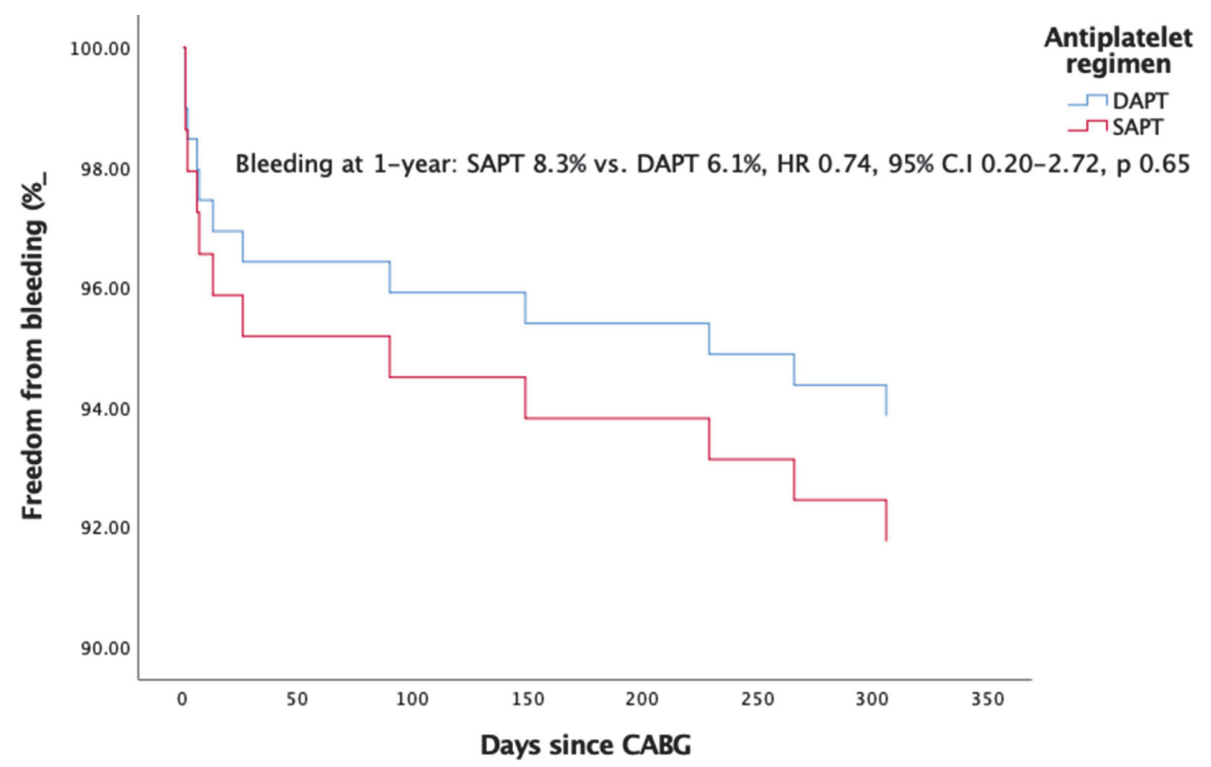

Abstract 60 Figure 1 Incidence of bleeding events at 1 year

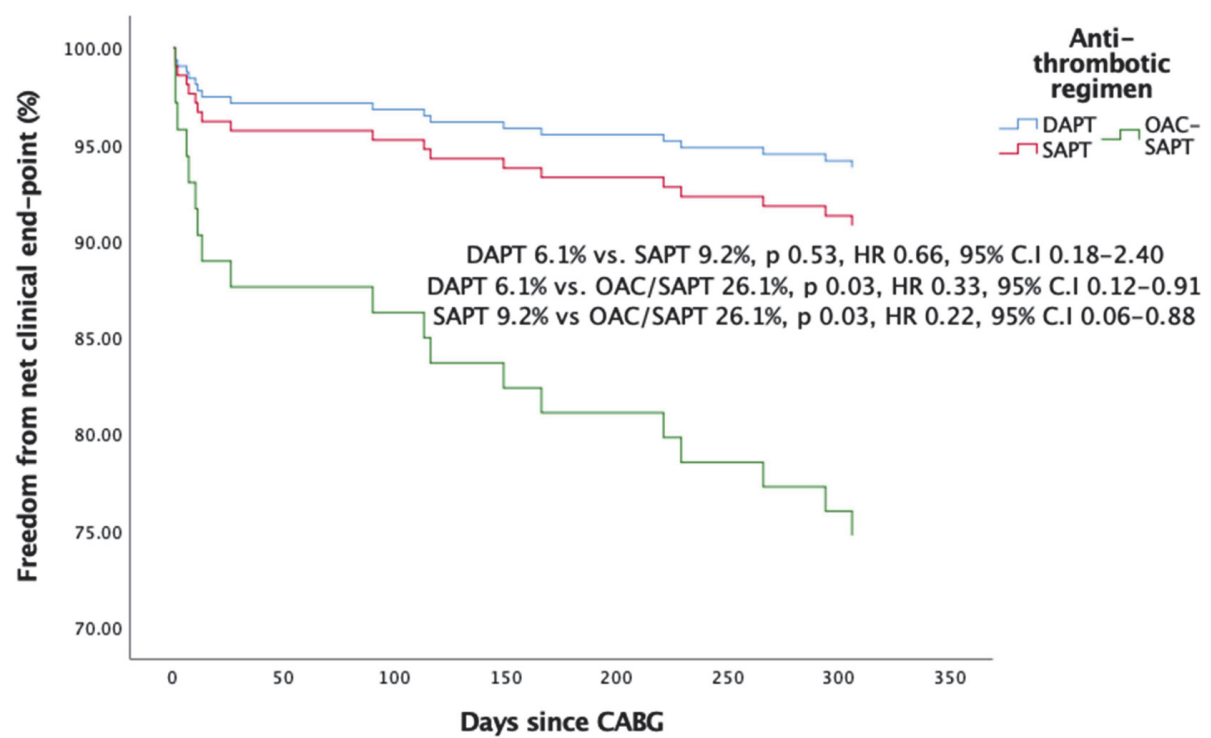

Abstract 60 Figure 2 Net clinical composite end-point at 1 year 
presenting with NSTEMI (1). We sought to ascertain adherence to current guidelines and compare outcomes with the use of different antithrombotic regimes in our institution.

Methods 388 patients underwent urgent coronary artery bypass grafting (CABG) in our institution between 2016 and 2018. The national electronic healthcare record was used to retrospectively collect data. Patients meeting the ESC universal definition of myocardial infarction at presentation were included. The primary safety outcome was defined as any Bleeding Academic Research Consortium Bleeding (BARC) Type 2-5 event on therapy at 1 year. The primary efficacy outcome was defined as a composite of non-fatal MI, CV death, unplanned revascularisation and documented graft failure at 1 year. The net clinical composite end point was a combination of both ischaemic and bleeding end points at 1 year.

Results 181 patients met our inclusion criteria (table 1). Mean age $=67.8 \pm 9.4$. Male sex $=80.6 \%$. Mean follow-up duration $=710 \pm 177$ days. $60.2 \%$ were managed with SAPT (aspirin $300 \mathrm{mg}$ OD), 27.1\% with DAPT (90\% aspirin $75 \mathrm{mg}$ OD plus clopidogrel $75 \mathrm{mg}$ OD) and $12.7 \%$ with oral anticoagulation (OAC) plus SAPT. OAC regimes were warfarin plus aspirin (60.9\%), NOAC plus aspirin (34.8\%) and NOAC plus clopidogrel (4.3\%). The primary safety end point was observed in $6.1 \%$ in the DAPT group, $9.2 \%$ in the SAPT group (HR 0.74; 95\% CI $0.20-2.72 ; \mathrm{p} 0.65$ ) and $21.7 \%$ in

\begin{tabular}{|c|c|c|c|c|}
\hline Characteristic & $\begin{array}{l}\text { SAPT } \\
(n=109)\end{array}$ & DAPT $(n=49)$ & $p$ value & $\begin{array}{l}\text { OAC plus } \\
\text { SAPT }(n=23)\end{array}$ \\
\hline Age [mean \pm SD] & $67.9 \pm 9.6$ & $65.7 \pm 9.1$ & 0.18 & $71.8 \pm 7.3$ \\
\hline Gender Male & $88(80.7 \%)$ & $40(81.6 \%)$ & 1.0 & $18(78.3 \%)$ \\
\hline Female & $21(19.3 \%)$ & $9(18.4 \%)$ & & $5(21.7 \%)$ \\
\hline Procedure $C A B G$ & $102(93.6 \%)$ & $48(98.0 \%)$ & & $22(95.7 \%)$ \\
\hline$C A B G \& A V R$ & $7(6.4 \%)$ & $1(2.0 \%)$ & & $1(4.3 \%)$ \\
\hline Diabetes & $40(36.7 \%)$ & $11(22.4 \%)$ & 0.08 & $12(52.2 \%)$ \\
\hline $\begin{array}{l}\text { eGFR }\left(\mathrm{ml} / \mathrm{min} / 1.73 \mathrm{~m}^{2}\right) \\
{[\text { mean } \pm \mathrm{SD}]}\end{array}$ & $55.2 \pm 11.2$ & $58.3 \pm 5.4$ & 0.11 & $53.3 \pm 9.4$ \\
\hline Troponin (ng/L) & 494.6 & $562.5 \pm 1048.1$ & 0.64 & 711.5 \\
\hline$\left[\right.$ mean $\left._{ \pm} \mathrm{SD}\right]$ & \pm 705.0 & & & \pm 1063.3 \\
\hline LVEF Normal & $64(58.7 \%)$ & $30(61.2 \%)$ & & $10(43.5 \%)$ \\
\hline Mild & $24(22.0 \%)$ & $5(10.2 \%)$ & & $9(39.1 \%)$ \\
\hline Moderate & $15(13.8 \%)$ & $6(12.3 \%)$ & & $1(4.3 \%)$ \\
\hline Severe & $6(5.5 \%)$ & $8(16.3 \%)$ & & $3(13.0 \%)$ \\
\hline
\end{tabular}

\begin{tabular}{lllll} 
Abstract 60 Table 2 & 1 year clinical end points \\
\hline End point & $\begin{array}{l}\text { SAPT } \\
(\mathrm{n}=109)\end{array}$ & $\begin{array}{l}\text { DAPT } \\
(\mathrm{n}=49)\end{array}$ & $\begin{array}{c}\text { p value } \\
\text { OAC plus } \\
\text { SAPT }(\mathrm{n}=23)\end{array}$ \\
\hline $\begin{array}{l}\text { Primary efficacy end point- no. (\%) } \\
\text { Non-fatal } \mathrm{MI}\end{array}$ & $2(1.8)$ & $0(0.0)$ & 1.0 & $1(4.3)$ \\
Unplanned revascularisation & $2(1.8)$ & $0(0.0)$ & & $1(4.3)$ \\
Graft failure & $2(1.8)$ & $0(0.0)$ & $1(4.3)$ \\
CV death & $2(1.8)$ & $0(0.0)$ & $1(4.3)$ \\
Ischaemic stroke- no. (\%) & $0(0.0)$ & $0(0.0)$ & $0(0.0)$ \\
Primary safety end point- no. (\%) & $2(1.8)$ & $2(4.1)$ & 0.59 & $1(4.3)$ \\
BARC 2-5 bleeding & $9(8.3)$ & $3(6.1)$ & 0.76 & $5(21.7)$ \\
Net clinical efficacy/safety & $10(9.2)$ & $3(6.1)$ & 0.53 & $6(26.1)$ \\
end-point- no. (\%) & & & & \\
\hline
\end{tabular}

the OAC plus SAPT group (table 2, figure 1). The primary efficacy end point occurred in $0.0 \%$ in the DAPT group, $1.8 \%$ in the SAPT group (p 1.0) and $4.3 \%$ in the OAC plus SAPT group. The net clinical efficacy/safety end point occurred in $6.1 \%$ in the DAPT group, $9.2 \%$ in the SAPT group (HR 0.66 ; 95\% CI $0.18-2.40 ; \mathrm{p} 0.53$ ) and $26.1 \%$ in the OAC plus SAPT group (figure 2). Ischaemic stroke occurred in $4.1 \%$ in the DAPT group vs $1.8 \%$ in the SAPT group (p 0.59).

Conclusions There was no statistically significant difference in either bleeding or ischaemic events between DAPT and SAPT. There was a trend towards reduction in both ischaemic and bleeding events with DAPT vs high dose SAPT. Our population size was underpowered to detect ischaemic events, however our findings would support the safety of current guidelines and advocate the use of DAPT following surgical revascularisation in NSTEMI.

A significantly higher rate of bleeding was observed in the OAC plus SAPT group. This population are not readily comparable to the other regimes in view of the underlying need for OAC for stroke prophylaxis. This does however raise the question about the potential safety of OAC monotherapy post $\mathrm{CABG}$ in patients with non-valvular $\mathrm{AF}$ as a method of bleeding risk reduction.

\section{THE EFFECT OF COVID-19 LOCKDOWN ON STEMI PRESENTATIONS: AN EARLY PHASE ANALYSIS}

${ }^{1} \mathrm{~N}$ Chongprasertpon, ${ }^{2} \mathrm{JJ}$ Coughlan, 'S Arockiam, 'S Arnous, 'T Kiernan. 'University Hospital Limerick, Limerick Ireland; ${ }^{2}$ German Heart Centre, Munich, Germany

\subsection{6/heartjnl-2020-ICS.61}

Introduction There is growing concern that public restrictions to minimize the transmission of the severe acute respiratory syndrome coronavirus 2 (SARS-CoV-2) causing coronavirus disease 2019 (COVID-19) as well as patients' concerns about developing the disease may be associated with delays in the presentation of ST elevation myocardial infarction (STEMI) with subsequent harmful effects on patient outcomes. In this study we sought to determine whether public restrictions implemented in the early phase of the COVID-19 lockdown was associated with any delay in patients presenting to medical attention and to assess for any associated difference in in-hospital mortality. STEMI patients were brought to the cardiac catheterisation laboratory and treated by healthcare workers utilising complete personal protective equipment (PPE). Therefore, we also aimed to determine if there was any increase in the cardiac catheterisation laboratory arrival to wire cross time.

Methods This was a retrospective observational analysis of all STEMI patients presenting to University Hospital Limerick during the early phase of the COVID-19 lockdown from March 27, 2020 to April 17, 2020. These data were compared to a reference period of data from March 27, 2019 to April 17, 2019. The data are expressed as both means and medians, with $95 \%$ confidence intervals of the difference between means and estimated effect sizes also calculated. The Independent Samples $t$ Test, Mann Whitney U Test and Fisher's Exact Test were utilized as appropriate.

Results The baseline demographics are demonstrated in table 1 and the outcomes of interest in table 2. The relative risk reduction of a patient presenting with STEMI during the 